\title{
AN ELEMENTARY GREEN IMPRIMITIVITY THEOREM FOR INVERSE SEMIGROUPS
}

\author{
BERNHARD BURGSTALLER
}

\begin{abstract}
A Morita equivalence similar to that found by Green for crossed products by groups will be established for crossed products by inverse semigroups. More precisely, let $G$ be an inverse semigroup, $H$ a finite sub-inverse semigroup of $G$ and $A$ a $G$-algebra or a $H$-algebra. Then the crossed product $A \rtimes H$ is Morita equivalent to a certain crossed product $B \rtimes G$.
\end{abstract}

\section{INTRODUCTION}

In a classical paper [8, Green showed that for a closed subgroup $H$ of a locally compact group $G$, and a $G$-algebra $A$ there exits a Morita equivalence between $A \rtimes H$ and $C_{0}(G / H, A) \rtimes G$ via an imprimitivity bimodule over these algebras ([8, Prop. 3]). This useful result was discussed and generalized in many directions, for example, in [16, 20, 1, 7].

In this note we shall establish an analogous imprimitivity theorem for an inverse semigroup $G$ and a finite sub-inverse semigroup $H \subseteq G$ for crossed products in Sieben's sense [19. As a corollary of this, we show this holds true also for a given $H$-algebra $A$, and thus this may be usefully combined with induction like in Kasparov [10, 9. Actually, this note was motivated by the fact that the BaumConnes map 2 for groups $G$ is a kind of extrapolation of Green-Julg isomorphisms for crossed products by $G$ of induced algebras by compact subgroups $H \subseteq G$, as noted by Meyer and Nest in 13. In establishing that, Kasparov's induction plays a fundamental role. To potentially carry this result over from groups to inverse semigroups, we need induction for compact (and thus finite) sub-inverse semigroups $H \subseteq G$, and this is now provided in this note. Actually, in the meanwhile we have made considerable progress in this direction and were able to establish a BaumConnes map for fibered $G$-algebras [5, 4, founding on this note.

We are going to give a brief summary of this article. At first we rewrite the inverse semigroup crossed product $A \rtimes H$ as a groupoid crossed product $A \rtimes \mathcal{G}$ to have a group-like construction. Then we adapt and follow Green's proof 8 , p. 199$204]$ in a natural way. The action on a certain quotient space $G_{\mathcal{G}} / \mathcal{G}(G / H$ in Green [8]) is similar to the regular representation action by Khoshkam and Skandalis [11. After establishing Green's imprimitivity Theorem 3.7, we apply it to the induced algebra (in the sense of Kasparov [10, 9]) $A$ of a $H$-algebra $D$, and restrict to ideals to get the second Green imprimitivity theorem, Corollary 3.9 .

1991 Mathematics Subject Classification. 46L55, 20M18, 46L08.

Key words and phrases. imprimitivity theorem, inverse semigroup, induction, crossed product, Morita equivalence. 


\section{Preparing DEFinitions AND CROSSED PRoducts}

We begin by recalling crossed products in the sense of Khoshkam and Skandalis [11 and Sieben [19, but use several notions from [6]. Let $G$ denote an inverse semigroup.

Definition 2.1. A $G$-algebra $A$ is a $C^{*}$-algebra $A$ endowed with a $G$-action in the following sense: there exists a semigroup homomorphism $\alpha: G \rightarrow \operatorname{End}(A)$, written as $g(a):=\alpha_{g}(a)$, such that

$$
g g^{*}(a) b=a g g^{*}(b)
$$

for all $a, b \in A$ and $g \in G$.

Such a $G$-algebra (whose definition is equivalent to [6, Def. 3.1]) is a special case of $G$-algebras in the sense of $[19$ and [11.

Definition 2.2. Let $\mathbb{F}(G, A)$, or $\mathbb{F}$ for brevity, be the universal $*$-algebra over $\mathbb{C}$ generated by disjoint copies of $A$ and $G$ subject to the relations that the $*$-algebraic relations of $A$ are respected, the multiplication and involution of $G$ are respected, and the relations

$$
g(a) g g^{*}=g a g^{*}, \quad g g^{*} a=a g g^{*}
$$

hold true for all $a \in A$ and $g \in G$. We shall identify $G$ and $A$ as subsets of $\mathbb{F}$. The algebraic crossed product $A \rtimes_{\text {alg }} G \subseteq \mathbb{F}$ denotes the linear span of all elements of the form $a g(a \in A, g \in G)$, which are usually denoted by $a \rtimes g$, and is a $*$-subalgebra of $\mathbb{F}$.

Definition 2.3. We denote by $G_{0} \subseteq G$ the idempotent elements of $G$, and by $E(G) \subseteq \mathbb{F}$ the set of all projections of the form $e_{0}\left(1-e_{1}\right) \ldots\left(1-e_{n}\right) \in \mathbb{F}$ with $e_{0}, \ldots, e_{n} \in G_{0}$ and $n \geq 0$. The subset $G_{E}:=\{g p \in \mathbb{F} \mid g \in G, p \in E(G)\} \subseteq \mathbb{F}$ is an inverse semigroup in $\mathbb{F}$ (under multiplication and taking adjoint). We even shall write $a \rtimes g:=a g$ when $a \in A$ and $g \in G_{E}$.

Note that then the identities

$$
a \rtimes g p=a g p=g g^{*} a g g^{*} g p=g g^{*}(a) g p=g g^{*}(a) \rtimes g p
$$

hold in $\mathbb{F}$ for all $a \in A$ and $g p \in G_{E}(g \in G, p \in E(G))$.

Definition 2.4. Hence, it is natural to call an expression $a \rtimes g p \in \mathbb{F}$ with $g \in$ $G, p \in E(G)$ and $a \in A_{g g^{*}}:=g g^{*}(A)$ to be standard.

The reader should be cautioned that the first relation of (2) is not true in general for $g \in G_{E}$ (consider for example $(1-e)(a)=0$ for the trivial $G$-action on $A$ ), however the second relation of (2) and identity (1) remain true for $g \in G_{E}$ (see Lemma 2.8 below).

The full crossed product $A \rtimes G$ is the closure of the image of $A \rtimes_{\text {alg }} G$ under the universal $*$-representation $\pi$ of $\mathbb{F}$ on Hilbert space ([11, Def. 5.4] or [6, 5.16, $6.2,8.4])$. It is easy to see with the reduced representations [11, p. 271] that $\pi$ is injective on $A \rtimes_{\text {alg }} G$, and so the latter is a pre- $C^{*}$-algebra with a $C^{*}$-norm. Sieben's crossed product $A \widehat{\rtimes} G$ is defined to be the image of $A \rtimes_{\text {alg }} G$ under the universal $*$-representation $\tau$ of $\mathbb{F}$ on Hilbert space satisfying $\tau\left(g(a)-g a g^{*}\right)=0$ (see [19]). We write $a \widehat{\rtimes} g$ for $\tau(a g)$. Note, in particular, that $\widehat{\rtimes}$ is compatible:

$$
e(a) \widehat{\rtimes} g=a \widehat{\rtimes} e g \quad \forall a \in A, g \in G_{E}, e \in E(G) .
$$


Notice that this identity is not true for $\rtimes$, and this compatibility is actually the essential difference between the full crossed product and Sieben's crossed product.

Definition 2.5. Let us now be given a finite sub-inverse semigroup $H^{\prime} \subseteq G$ of $G$. Denote by $H$ the (finite) groupoid associated to $H^{\prime}$ (cf. [14]). More precisely, let $H^{(0)} \subseteq \mathbb{F}$ be the set of all nonzero minimal projections of $E\left(H^{\prime}\right)$ and

$$
H=\left\{t e \in \mathbb{F} \mid t \in H^{\prime}, e \in H^{(0)}\right\} \backslash\{0\} \quad \subseteq \mathbb{F} .
$$

The multiplication within $H$ is that inherited from $\mathbb{F}$.

Definition 2.6. Define

$$
G_{H}=\left\{g e \in \mathbb{F} \mid g \in G, e \in H^{(0)}, g^{*} g \geq e\right\} \backslash\{0\} \quad \subseteq \mathbb{F} .
$$

We endow $G_{H}$ with an equivalence relation: $g \equiv h$ if and only if there exists $t \in H$ such that $g t=h\left(g, h \in G_{H}\right)$. We denote by $G_{H} / H$ the set-theoretical quotient of $G_{H}$ by $\equiv$.

We shall exclusively work with representatives in this quotient; writing $g \in$ $G_{H} / H$ means implicitly that $g \in G_{H}$ and we use no class brackets; if then $g \in G_{H}$ is meant or the class $g \in G_{H} / H$ becomes apparent from the context. For an assertion $\mathcal{A}$ we let $[\mathcal{A}]$ be the real number 0 if $\mathcal{A}$ is false, and 1 if $\mathcal{A}$ is true.

Definition 2.7. Let $C_{0}\left(G_{H} / H\right)$ denote the commutative $C^{*}$-algebra of (continuous) complex-valued functions vanishing at infinity of the (discrete) set $G_{H} / H$ with the pointwise operations. The delta function $\delta_{g}$ in $C_{0}\left(G_{H} / H\right)$ is denoted by $g\left(g \in G_{H} / H\right)$. The algebra $C_{0}\left(G_{H} / H\right)$ is endowed with the $G$-action $g(h):=\left[g h \in G_{H}\right] g h$, where $g \in G$ and $h \in G_{H} / H$ (of course, $g h \in G_{H}$ is equivalent to $\left.g^{*} g \geq h h^{*}\right)$. We let $A \otimes C_{0}\left(G_{H} / H\right)$ be the $C^{*}$-algebraic tensor product endowed with the diagonal action by $G$.

Lemma 2.8. (i) If $g_{1}, \ldots, g_{n} \in G_{H}$ are mutually different then $\sum_{i=1}^{n} a_{i} \rtimes g_{i}=$ 0 (sum of standard elements) implies $a_{1}=\ldots=a_{n}=0$.

(ii) The $G$-action on a $G$-algebra $A$ extends naturally to an inverse semigroup $G_{E}$-action on $A$ (i.e. one sets $(1-e)(a):=a-e(a)$ for all $a \in A$ and $e \in E)$.

(iii) The formulas $(a \rtimes g)(b \rtimes h)=a g(b) \rtimes g h$ and $(b \rtimes h)^{*}=h^{*}\left(b^{*}\right) \rtimes h^{*}$ hold in $\mathbb{F}$ for all $g, h \in G_{E}, a \in A_{g g^{*}}:=g g^{*}(A)$ and $b \in A$.

Proof. (i) We may assume that all $g_{i}$ have the same source projection in $H^{(0)}$ (otherwise multiply $\sum a_{i} \rtimes g_{i}$ from the left with each single minimal mutually orthogonal projection of $\left.H^{(0)}\right)$. Hence we may fix $e_{1}, \ldots, e_{m} \in G_{0}$ such that for all $1 \leq i \leq n$ we have $g_{i}=h_{i}\left(1-e_{1}\right) \ldots\left(1-e_{m}\right)$ for certain mutually different $h_{i} \in G$. Expanding, we get $0=\sum_{i=1}^{n} a_{i} g_{i}=\sum_{i=1}^{n} a_{i} h_{i}-\sum_{i=1}^{n} a_{i} h_{i} e_{1} \pm \ldots$ in $\mathbb{F}$. Now note that by the reduced representation of the algebraic crossed product $A \rtimes_{\text {alg }} G$ in [11, p. 271] the elements $h_{i}, h_{j} e_{1}$ et cetera in the last sum are linearly independent, as far as they are all different. Certainly, however, we may conclude that $a_{i} h_{i}=0$ for all $1 \leq i \leq n$, because assuming that $h_{i}=h_{j} e_{k_{1}} \ldots e_{k_{s}}$ would yield $g_{i}=h_{j} e_{k_{1}} \ldots e_{k_{s}}\left(1-e_{1}\right) \ldots\left(1-e_{m}\right)=0$ (however $\left.0 \notin G_{H}\right)$. This yields the claim.

(ii) By the linear independence of the elements of $G_{0}$, it easy to see that we have already a well-defined semigroup homomorphism $\alpha: E(G) \rightarrow \operatorname{End}(A)$ defined by $\alpha_{e}(a)=e(a)$ and $\alpha_{1-e}(a)=a-e(a)$ for all $a \in A$ and $e \in G_{0}$. To extend it to 
$G_{E}$, we consider an ambiguous representation $0 \neq g p=h q \in G_{E}$ for some $h, g \in G$ and $p, q \in E(G)$. Then $g p q=h p q$ and so $g=h$ by a similar argument as in (i). Thus $g^{*} g p=g^{*} g q$ in $E(G)$. Hence, the definition $\alpha_{g p}:=\alpha_{g} \alpha_{p}=\alpha_{g} \alpha_{g g^{*} p}=\alpha_{h} \alpha_{q}$ is well-defined ( $\alpha_{g}$ denotes the given $G$-action).

(iii) Let $g, h \in G, p, q \in E(G), a \in A_{g p g^{*}}$ and $b \in A$. We have, for example, by (11) for the extended action of (ii), (2) and because $a=g p g^{*}(a) \in A_{g p g^{*}}$ that

$$
a g p \cdot b h q=g p g^{*}(a) \cdot g p \cdot b \cdot h q=g p g^{*}(a) \cdot g(b) \cdot g p \cdot h q=a \cdot g p(b) \cdot g p h q
$$

in $\mathbb{F}$.

Lemma 2.9. If $A$ is a $G$-algebra and $I \subseteq A$ a $G$-invariant ideal in $A$ then $I \rtimes G \subseteq$ $A \rtimes G$ and $I \widehat{\rtimes} G \subseteq A \widehat{\rtimes} G$ canonically.

Proof. A representation of $\mathbb{F}(G, I)$ is given by a covariant triple $(\sigma, U, H)$ for some Hilbert space $H$, a $*$-homomorphism $\sigma: I \rightarrow B(H)$ and an inverse semigroup homomorphism $U: G \rightarrow B(H)$ satisfying the analogous defining relations as in (2). Proceed as in 3, Lemma A.4] to show that the representation of $\mathbb{F}(G, I)$ extends to $\mathbb{F}(G, A)$. Hence $\overline{\mathbb{F}(G, I)} \supseteq I \rtimes G \rightarrow A \rtimes G \subseteq \overline{\mathbb{F}(G, A)}$ is isometric.

\section{THE IMPRIMITIVITY THEOREM}

We shall now introduce an imprimitivity bimodule in the sense of Rieffel [17].

Definition 3.1. We introduce the spaces

$$
\begin{aligned}
& B_{0}=A \rtimes_{\text {alg }} H:=\operatorname{span}\left\{a \rtimes t \in A \rtimes_{\text {alg }} G \mid a \in A_{t t^{*}}, t \in H\right\}, \\
& X_{0}=\operatorname{span}\left\{a \rtimes g \in A \rtimes_{\text {alg }} G \mid a \in A, g \in G_{H}\right\}, \\
& E_{0}=\left(A \otimes C_{0}\left(G_{H} / H\right)\right) \rtimes_{\text {alg }} G .
\end{aligned}
$$

The spaces $B_{0} \subseteq A \rtimes G$ and $E_{0}$ are regarded as pre- $C^{*}$-algebras. We make $X_{0}$ to a right pre-Hilbert module over $B_{0}$ (cf. [17, Def. 2.8]) by the following operations

$$
\begin{array}{ll}
X_{0} \times B_{0} \longrightarrow X_{0}: & (a \rtimes g)(c \rtimes t):=a g(c) \rtimes g t, \\
X_{0} \times X_{0} \longrightarrow B_{0}: & \langle a \rtimes g, b \rtimes h\rangle_{B_{0}}:=\left[g^{*} h \in H\right] g^{*}\left(a^{*} b\right) \rtimes g^{*} h
\end{array}
$$

for $a, b \in A, c \in A_{t t^{*}}, g, h \in G_{H}$ and $t \in H$, and to a left pre-Hilbert module over $E_{0}$ by

$$
\begin{array}{ll}
E_{0} \times X_{0} \longrightarrow X_{0}: & (a \otimes r \rtimes s)(b \rtimes j):=\left[s j \in G_{H}\right][r \equiv s j] a s(b) \rtimes s j, \\
X_{0} \times X_{0} \longrightarrow E_{0}: & \langle a \rtimes g, b \rtimes h\rangle_{E_{0}}:=a g h^{*}\left(b^{*}\right) \otimes g \rtimes g h^{*}
\end{array}
$$

for $a, b \in A, r \in G_{H} / H, s \in G$ and $j, g, h \in G_{H}$.

Because of identity (3) we may write every element in an algebraic crossed product as the sum of standard elements. Because of the linear independence statement of Lemma 2.8. (i) we may then extend the formulas of Definition 3.1 for standard expressions by linearity. We need however remark, that

Lemma 3.2. The formulas of Definition 3.1 remain however valid also for nonstandard expressions as stated.

Proof. For example, by considering the formula of the map $E_{0} \times X_{0} \rightarrow X_{0}$, given elementary elements $a \otimes r \rtimes s s^{*} s \in E_{0}$ and $b \rtimes j p \in X_{0}$ with $a \in A, r \in G_{H} / H, s, j \in$ 
$G$ and $s^{*} s, p \in E(G)$, we go over to their standard form $\left[s s^{*} r \in G_{H}\right] s s^{*}(a) \otimes s s^{*} r \rtimes$ $s \in E_{0}$ and $j j^{*}(b) \rtimes j p \in X_{0}$ according to (3). Then their module product in $X_{0}$ is

$$
\begin{aligned}
& {\left[s s^{*} r \in G_{H}\right]\left[s j p \in G_{H}\right]\left[s s^{*} r \equiv \operatorname{sjp}\right] s s^{*}(a) s\left(j j^{*}(b)\right) \rtimes s j p } \\
= & {\left[\operatorname{sjp} \in G_{H}\right][r \equiv \operatorname{sjp}] \operatorname{sjj}^{*} s^{*}(a) s j j^{*} s^{*} s(b) \rtimes s j p } \\
= & {\left[\operatorname{sjp} \in G_{H}\right][r \equiv \operatorname{sjp}] a s(b) \rtimes \operatorname{sjp} }
\end{aligned}
$$

by (11), (3) and because $\left[s s^{*} r \in G_{H}\right]$ cancels because $s s^{*} r \equiv s j p$ implies $s s^{*} r=s j p t$ for some $t \in H^{(0)}$, implies $s s^{*} r=r$ (since the source projection of $s s^{*} r=s j p t \in G_{H}$ is in $H^{(0)}$ and thus cannot become smaller than the one of $r \in G_{H}$ ), implies $s s^{*} r=r \in G_{H}$. This is the same element as taking the module product formula in $X_{0}$ for the given non-standard elements $a \otimes r \rtimes s \in E_{0}$ and $b \rtimes j p \in X_{0}$. The above formulas for non-standard expressions can then be extended also linearly.

Proposition 3.3. Straightforward, but again rather time-consuming, extensive and tedious computations show that we have

$$
\begin{aligned}
& \langle x, y b\rangle_{B_{0}}=\langle x, y\rangle_{B_{0}} b, \quad\langle x, y\rangle_{B_{0}}^{*}=\langle y, x\rangle_{B_{0}}, \\
& \langle f x, y\rangle_{E_{0}}=f\langle x, y\rangle_{E_{0}}, \quad\langle x, y\rangle_{E_{0}}^{*}=\langle y, x\rangle_{E_{0}}, \\
& \langle f x, y\rangle_{B_{0}}=\left\langle x, f^{*} y\right\rangle_{B_{0}}, \quad\langle x, y b\rangle_{E_{0}}=\left\langle x b^{*}, y\right\rangle_{E_{0}}, \quad x\langle y, z\rangle_{B_{0}}=\langle x, y\rangle_{E_{0}} z
\end{aligned}
$$

for all $x, y, z \in X_{0}, b \in B_{0}$ and $f \in E_{0}$ (cf. [17, Def. 6.10]).

Proof. For convenience of the reader we demonstrate the first identity of line (5), which is the most difficult of all of these, and the others should not present further new difficulties (for the *-algebraic operations in $B_{0}$ use the formulas of Lemma 2.8. (iii)). We have

$$
\begin{aligned}
& \langle(a \otimes r \rtimes s)(b \rtimes g), c \rtimes h\rangle_{B_{0}} \\
= & {\left[s g \in G_{H}\right][r \equiv s g]\langle a s(b) \rtimes s g, c \rtimes h\rangle_{B_{0}} } \\
= & {\left[s g \in G_{H}\right][r \equiv s g]\left[g^{*} s^{*} h \in H\right] g^{*} s^{*}\left(s\left(b^{*}\right) a^{*} c\right) \rtimes g^{*} s^{*} h }
\end{aligned}
$$

for $a, b, c \in A, r \in G_{H} / H, s \in G$ and $g, h \in G_{H}$, and

$$
\begin{aligned}
& \left\langle b \rtimes g,(a \otimes r \rtimes s)^{*}(c \rtimes h)\right\rangle_{B_{0}} \\
= & \left\langle b \rtimes g,\left(\left[s^{*} r \in G_{H}\right] s^{*}\left(a^{*}\right) \otimes s^{*} r \rtimes s^{*}\right)(c \rtimes h)\right\rangle_{B_{0}} \\
= & {\left[s^{*} r \in G_{H}\right]\left[s^{*} h \in G_{H}\right]\left[s^{*} r \equiv s^{*} h\right]\left\langle b \rtimes g, s^{*}\left(a^{*}\right) s^{*}(c) \rtimes s^{*} h\right\rangle_{B_{0}} } \\
(7)= & {\left[g^{*} s^{*} h \in H\right]\left[s^{*} r \equiv s^{*} h\right]\left[s^{*} h \in G_{H}\right]\left[s^{*} r \in G_{H}\right] g^{*}\left(b^{*} s^{*}\left(a^{*}\right) s^{*}(c)\right) \rtimes g^{*} s^{*} h . }
\end{aligned}
$$

We only need to show that the scalar coefficients of the expressions (6) and (7) coincide, because observe that the vector parts coincides by a single application of identity (11). Note that the scalar $\left[g^{*} s^{*} h \in H\right]$ appears both in (6) and (7). Suppose that (6) is nonzero. Then $s g \in G_{H}$, and thus $s^{*} s \geq g g^{*}$ because $g \in G_{H}$ and $s g \in G_{H}$ (the source projection of $g$ is in $H^{(0)}$ and cannot be made smaller in $s g$ ). On the other hand, $r \equiv s g$ and so there exists some $t \in H$ such that $r=s g t$. Hence, $s^{*} r=s^{*} s g t=g t \in G_{H}$. Thus $\left[s^{*} r \in G_{H}\right]$ appearing in (7) is nonzero. Since $g^{*} s^{*} h \in H$, both its source and range projection are in $H^{(0)}$. Hence, since $h \in G_{H}, s^{*} h$ must be in $G_{H}$ too in order not to loose information on the source projection of $s^{*} h$. Since $g \in G_{H}$, the source projection of $g^{*}$ and the range projection of $s^{*} h$ must perfectly fit together such that $g^{*} s^{*} h \in H$. But this implies that $g \cdot\left(g^{*} s^{*} h\right)=s^{*} h$ and hence $g \equiv s^{*} h$. This implicates $s^{*} r \equiv g \equiv s^{*} h$. 
We have obtained that $\left[s^{*} r \equiv s^{*} h\right]\left[s^{*} h \in G_{H}\right]$ appearing in (7) is nonzero. Hence (77) is nonzero. In the other direction suppose that (77) is nonzero. Since $g^{*} s^{*} h \in H$ and $g, h \in G_{H}$, we can completely analogously deduce as before that $s g \equiv h$. This already implies that $\left[s g \in G_{H}\right]$ appearing in (6) is nonzero. Since $s^{*} h, s^{*} r \in G_{H}$ and $h, r \in G_{H}$, we get $s s^{*} \geq h h^{*}, r r^{*}$. Hence $s^{*} r \equiv s^{*} h$ implies $r \equiv h \equiv s g$. Thus (66) is nonzero.

Lemma 3.4. The inner products of $X_{0}$ are positive.

Proof. Let $\left(a_{\alpha}\right)_{\alpha}$ be an approximate identity of $A$. Let $x=\sum_{s=1}^{m} b_{s} \rtimes h_{s}$ in $X_{0}$ and choose for every different equivalence class $h_{s} H$ in $G_{H} / H$ exactly one representative $g_{i}:=h_{s} \in G_{H}$, where $1 \leq i \leq n$ with $n \leq m$. (We have a new index $i$ because for $h_{s_{1}} H=h_{s_{2}} H$ we would choose a common $g_{i}$.) Set $x_{i, \alpha}=a_{\alpha} \rtimes g_{i} \in X_{0}$. Set $x_{\alpha}=\sum_{i=1}^{n}\left\langle x_{i, \alpha}, x_{i, \alpha}\right\rangle_{E_{0}} x \in X_{0}$. Then

$$
\begin{aligned}
x_{\alpha} & =\sum_{i=1}^{n} \sum_{s=1}^{m}\left(a_{\alpha} g_{i} g_{i}^{*}\left(a_{\alpha}^{*}\right) \otimes g_{i} \rtimes g_{i} g_{i}^{*}\right)\left(b_{s} \rtimes h_{s}\right) \\
& =\sum_{i, s}\left[g_{i} g_{i}^{*} h_{s} \in G_{H}\right]\left[g_{i} \equiv g_{i} g_{i}^{*} h_{s}\right] a_{\alpha} g_{i} g_{i}^{*}\left(a_{\alpha}^{*}\right) g_{i} g_{i}^{*}\left(b_{s}\right) \rtimes g_{i} g_{i}^{*} h_{s} \\
& =\sum_{i, s}\left[g_{i} \equiv h_{s}\right] a_{\alpha} g_{i} g_{i}^{*}\left(a_{\alpha}^{*}\right) g_{i} g_{i}^{*}\left(b_{s}\right) \rtimes g_{i} g_{i}^{*} h_{s} \\
& =\sum_{s=1}^{m} h_{s} h_{s}^{*}\left(a_{\alpha} a_{\alpha}^{*}\right) b_{s} \rtimes h_{s}
\end{aligned}
$$

where identity (8) follows from the fact that $g_{i} g_{i}^{*} h_{s} \in G_{H}$ and $h_{s} \in G_{H}$ implies $g_{i} g_{i}^{*} \geq h_{s}$, and so $g_{i} \equiv g_{i} g_{i}^{*} h_{s}$ implies $\left[g_{i} \equiv h_{s}\right] \neq 0$, which, on the other hand, implies $g_{i} g_{i}^{*}=h_{s} h_{s}^{*}$ and thus $\left[g_{i} g_{i}^{*} h_{s} \in G_{H}\right]\left[g_{i} \equiv g_{i} g_{i}^{*} h_{s}\right] \neq 0$. Identity (9) follows because we have chosen for each $h_{s}$ one but at most one equivalent $g_{i}$. We used also (11) and Lemma 2.8 (ii) there. Also (11) is used to easily compute that $\left\langle x, x-x_{\alpha}\right\rangle_{B_{0}} \rightarrow$ 0 . Consequently,

$$
\begin{aligned}
\langle x, x\rangle_{B_{0}} & =\lim _{\alpha}\left\langle x, x_{\alpha}\right\rangle_{B_{0}}=\lim _{\alpha} \sum_{i=1}^{n}\left\langle x,\left\langle x_{i, \alpha}, x_{i, \alpha}\right\rangle_{E_{0}} x\right\rangle_{B_{0}} \\
& =\lim _{\alpha} \sum_{i=1}^{n}\left\langle x, x_{i, \alpha}\right\rangle_{B_{0}}\left\langle x, x_{i, \alpha}\right\rangle_{B_{0}}^{*} \geq 0
\end{aligned}
$$

as in Green 8, page 202, with the last identity of (5). The argument for the positivity of $\langle x, x\rangle_{E_{0}}$ is similar. Here we choose, for example, $x_{\alpha}=x \sum_{e \in H^{(0)}}\left\langle a_{\alpha} \rtimes\right.$ $\left.e, a_{\alpha} \rtimes e\right\rangle_{B_{0}}$.

Proposition 3.5. We have the inequalities

$$
\langle f x, f x\rangle_{B_{0}} \leq\|f\|_{E_{0}}^{2}\langle x, x\rangle_{B_{0}}, \quad\langle x b, x b\rangle_{E_{0}} \leq\|b\|_{B_{0}}^{2}\langle x, x\rangle_{E_{0}}
$$

for all $x \in X_{0}, f \in E_{0}$ and $b \in B_{0}$ (cf. [17, Def. 6.10]).

Proof. For the proof of the first inequality regard $X_{0}$ as an pre-Hilbert $B_{0}$-module. Let $\mathcal{M}(A)$ denote the multiplier algebra of $A$. For a nonzero standard element $f=a \otimes r \rtimes s \in E_{0}$ we compute $f^{*} f$ with the formulas of Lemma 2.8. (iii), and for 
another $x \in X_{0}$ we obtain, with Proposition 3.3 .

$$
\begin{aligned}
& \|f\|_{E_{0}}^{2}\langle x, x\rangle_{B_{0}}-\langle f x, f x\rangle_{B_{0}}=\left\langle\left(\|f\|_{E_{0}}^{2}-f^{*} f\right) x, x\right\rangle_{B_{0}} \\
= & \left\langle\left(\|f\|_{E_{0}}^{2}-s^{*}\left(a^{*} a\right) \otimes s^{*} r \rtimes s^{*} s\right) x, x\right\rangle_{B_{0}} \\
= & \langle z x, z x\rangle_{B_{0}}+\langle(1-p) x,(1-p) x\rangle_{B_{0}} \geq 0,
\end{aligned}
$$

where $z:=\left(\|f\|_{E_{0}}^{2}-s^{*}\left(a^{*} a\right)\right)^{1 / 2} \otimes s^{*} r \rtimes s^{*} s$ and $p:=\|f\|_{E_{0}}^{2} \otimes s^{*} r \rtimes s^{*} s$ are elements in $\left(\mathcal{M}(A) \otimes C_{0}\left(G_{H} / H\right)\right) \rtimes G$. Of course, we had here temporarily to replace our coefficient algebra $A$ by $\mathcal{M}(A)$ in order to include $\|f\|_{E_{0}}$ and have therefore some slightly larger new $E_{0}$. (Note that for general $f \in E_{0},\left(\|f\|_{E_{0}}^{2}-f^{*} f\right)^{1 / 2}$ need not be in $E_{0}$ and that is why we need to consider elementary elements $f$.)

By applying the norm $\|\cdot\|_{B_{0}}$ in $B_{0}$ to this inequality, we obtain $\|f x\| \leq\|f\|_{E_{0}}\|x\|$ (where $\|x\|:=\left\|\langle x, x\rangle_{B_{0}}\right\|^{1 / 2}$ ) for such elementary elements $f \in E_{0}$, and by taking sums of such elements we readily obtain $\|f x\| \leq\|f\|_{\ell^{1}\left(G, A \otimes C_{0}\left(G_{H} / H\right)\right)}\|x\|$ for all $f \in$ $E_{0}$. Hence, the $E_{0}$-module multiplication on $X_{0}$ is a $*$-homomorphism $E_{0} \rightarrow \mathcal{L}\left(X_{0}\right)$ which is an $\ell^{1}$-contractive representation into a pre- $C^{*}$-algebra. Since by definition the $C^{*}$-norm closure of $E_{0}$ is the enveloping $C^{*}$-algebra of $\ell^{1}\left(G, A \otimes C_{0}\left(G_{H} / H\right)\right)$ (cf. [11]) and so induced by the sum over all $\ell^{1}$-contractive representations, we must get $\|f\|_{\mathcal{L}\left(X_{0}\right)} \leq\|f\|_{E_{0}}$. It is well known from the theory of Hilbert-modules that one has $\langle f x, f x\rangle_{B_{0}} \leq\|f\|_{\mathcal{L}\left(X_{0}\right)}^{2}\langle x, x\rangle_{B_{0}}$ for adjoint-able operators $f$ (see for instance Lance [12, Prop. 1.2), and hence the first inequality of (10). The second inequality of (10) is proved similarly (but is easier as $B_{0}$ is even norm-closed).

Definition 3.6. Denote by $E_{X} \subseteq \overline{E_{0}}$ the norm closure of $\left\langle X_{0}, X_{0}\right\rangle_{E_{0}}$ under the $C^{*}$-norm $\|\cdot\|_{E_{0}}$, and by $B_{X} \subseteq \overline{B_{0}}$ the norm closure of $\left\langle X_{0}, X_{0}\right\rangle_{B_{0}}$ under the $C^{*}$ norm $\|\cdot\|_{B_{0}}$. We now apply the argument following [18, Prop. 3.1] to see that $X_{0}$ may be completed in semi-norm $\|x\|=\left\|\langle x, x\rangle_{B_{0}}\right\|^{1 / 2}$ (after factoring out the elements of norm 0 ) to obtain an $E_{X}-B_{X}$ imprimitivity bimodule $X$.

Theorem 3.7. Let $H^{\prime}$ be a finite sub-inverse semigroup of an inverse semigroup $G$ and denote by $H$ its associated finite groupoid (Definition 2.5). Let $A$ be a $G$ algebra. Then we have a $C^{*}$-algebraic Morita equivalence

$$
C_{0}\left(G_{H} / H, A\right) \widehat{\rtimes} G \quad \sim_{M} \quad A \widehat{\rtimes} H^{\prime}
$$

via the $E_{X}-B_{X}$ imprimitivity bimodule $X$ and isomorphisms $E_{X} \cong$ $C_{0}\left(G_{H} / H, A\right) \widehat{\rtimes} G$ and $B_{X} \cong A \widehat{\rtimes} H^{\prime}$.

Proof. The finite dimensional $C^{*}$-algebra $B_{X}=B_{0}$ is canonically isomorphic to the groupoid crossed product $A \rtimes H$, which is canonically isomorphic to the inverse semigroup crossed product $A \widehat{\rtimes} H^{\prime}$ by [15, Thm. 7.2]. To meet exactly the assumptions in [15], switch to the carrier algebra $\tilde{A}=p(A)$ for $p=\sum_{e \in H^{(0)}} e$ of $A$, which does not change the crossed product, that is, $\tilde{A} \widehat{\rtimes} H^{\prime}=A \widehat{\rtimes} H^{\prime}$.

Denote by $C_{0}\left(G_{H} / H, A\right) \subseteq A \otimes C_{0}\left(G_{H} / H\right)$ the norm closure of the linear span of

$$
\left\{a \otimes r \in A \otimes C_{0}\left(G_{H} / H\right) \mid a \in A_{r r^{*}}, r \in G_{H} / H\right\} .
$$

Note that $C_{0}\left(G_{H} / H, A\right)$ is a $G$-invariant ideal in $A \otimes C_{0}\left(G_{H} / H\right)$ and so

$$
C_{0}\left(G_{H} / H, A\right) \widehat{\rtimes} G \subseteq\left(A \otimes C_{0}\left(G_{H} / H\right)\right) \widehat{\rtimes} G
$$


embeds by Lemma 2.9. Using (11), let

$$
\sigma: E_{X} \longrightarrow C_{0}\left(G_{H} / H, A\right) \widehat{\rtimes} G: \sigma(a \otimes r \rtimes g)=a \otimes r \widehat{\rtimes} g
$$

be the canonical map $\left(a \in A, r \in G_{H} / H\right.$ and $g \in G$ ). (Note that there is always a canonical map $A \rtimes G \rightarrow A \widehat{\rtimes} G$.) It is surjective, because given a nonzero elementary element $a a^{*} \otimes r \widehat{\rtimes} g$ in $C_{0}\left(G_{H} / H, A\right) \widehat{\rtimes} G$ with $a \in A_{r r^{*}}, r \in G_{H} / H$ and $g \in G$ we note that

$$
a a^{*} \otimes r \widehat{\rtimes} g=r r^{*}\left(a a^{*} \otimes r\right) \widehat{\rtimes} g g^{*} g=g g^{*}\left(a a^{*}\right) \otimes g g^{*} r \widehat{\rtimes} r r^{*} g
$$

by the permeability (compatibility) of $\widehat{\rtimes}$ for projections in $E(G)$, see (4), so that we may assume that the given element $a a^{*} \otimes r \widehat{\rtimes} g$ satisfies $a \in A_{r r^{*}}, r \in G_{H} / H$ and $g \in G_{E}$ with $r r^{*}=g g^{*}$. Hence we get

$$
a a^{*} \otimes r \widehat{\rtimes} g=\sigma\left(\left\langle a \rtimes r, g^{*}(a) \rtimes g^{*} r\right\rangle_{E_{0}}\right) .
$$

If $\sigma$ were not injective, then its kernel $J \subseteq E_{X}$ were nonzero, and so would correspond to a nonzero ideal $I$ in $B_{X}$ via the $E_{X}-B_{X}$ imprimitivity module $X$ (see [18, Cor. 3.1]), which then would contain a nonzero element of the form $a \rtimes e \in I$ with $e \in H^{(0)}$. A nonzero element of the form $f=\langle a \rtimes e, a \rtimes e\rangle_{E_{0}}$ would be in $J$, however $\sigma$ is nonzero on $f$. We have obtained our result.

Definition 3.8. Now assume that $D$ is a $H^{\prime}$-algebra. Define, similarly as in [10, $\S 5$ Def. 2],

$$
\begin{gathered}
\operatorname{Ind}_{H^{\prime}}^{G}(D):=\left\{f: G_{H} \rightarrow D \mid \forall g \in G_{H}, t \in H \text { with } g t \in G_{H}: f(g t)=t^{*}(f(g)),\right. \\
\left.\|f(g)\| \rightarrow 0 \text { for } g H \rightarrow \infty \text { in } G_{H} / H\right\} .
\end{gathered}
$$

It is a $C^{*}$-algebra under the pointwise operations and the supremum's norm and becomes a $G$-algebra under the $G$-action $(g f)(h):=\left[g^{*} h \in G_{H}\right] f\left(g^{*} h\right)$ for $g \in G$, $h \in G_{H}$ and $f \in \operatorname{Ind}_{H^{\prime}}^{G}(D)$.

Corollary 3.9. Let $H^{\prime} \subseteq G$ be a finite sub-inverse semigroup of an inverse semigroup $G$. Let $D$ be a $H^{\prime}$-algebra. Then we have a $C^{*}$-algebraic Morita equivalence

$$
\operatorname{Ind} d_{H^{\prime}}^{G}(D) \widehat{\rtimes} G \quad \sim_{M} \quad D \widehat{\rtimes} H^{\prime} .
$$

Proof. Let $A$ denote $\operatorname{Ind}_{H^{\prime}}^{G}(D)$. Consider the $H^{\prime}$-invariant ideal $A_{0}$ of $A$ consisting of all functions which vanish outside $H$. Let us again view $B_{X}$ as $B_{X}=A \rtimes H$ as in the last proof before. Then $A_{0} \rtimes H$ embeds canonically as an ideal $J$ in $A \rtimes H=B_{X}$, and by [18, Cor. 3.1], associated to $J$ is the submodule in $X$ generated by

$$
Y_{0}=\left\{y \in X_{0} \mid\langle y, y\rangle_{B_{X}} \in J\right\}=\operatorname{span}\left\{g(a) \rtimes g \in X_{0} \mid a \in A_{0}, g \in G_{H}\right\} \subseteq X,
$$

and the ideal $I$ in $E_{X}$ generated by (and actually norm closure of)

$$
\left\langle Y_{0}, Y_{0}\right\rangle_{E_{X}}=\operatorname{span}\left\{g(a) \otimes g \widehat{\rtimes} g h^{*} \in E_{X} \mid a \in A_{0}, g, h \in G_{H}\right\} \subseteq E_{X} .
$$

Note that we are identifying $E_{X} \cong C_{0}\left(G_{H} / H, A\right) \widehat{\rtimes} G$ by the isomorphism $\sigma$ stated in (12). Using Lemma 2.9 the ideal $I$ is canonically isomorphic to $K \widehat{\rtimes} G \subseteq$ $E_{X}$, where $K$ denotes the $G$-invariant ideal in $C_{0}\left(G_{H} / H, A\right)$ which is the norm closure of the linear span of

$$
\left\{g(a) \otimes g \in C_{0}\left(G_{H} / H, A\right) \mid a \in A_{0}, g \in G_{H} / H\right\} .
$$

To see that the identical embedding $I \rightarrow K \widehat{\rtimes} G$ is surjective, write a given nonzero element $g(a) \otimes g \widehat{\rtimes} s \in K \widehat{\rtimes} G\left(a \in A_{0}, g \in G_{H} / H\right.$ and $\left.s \in G\right)$ as

$$
s s^{*} g(a) \otimes s s^{*} g \widehat{\rtimes} s s^{*} g g^{*} s \quad \in I
$$


with $s s^{*} g, s^{*} g \in G_{H}$ by the compatibility of $\widehat{\rtimes}$, see (4). We have a $G$-equivariant isomorphism $\psi: A \rightarrow K$ defined by

$$
\psi(f)=\left.\sum_{g \in G_{H} / H} f\right|_{g} \otimes g=\sum_{g \in G_{H} / H} g\left(\left.g^{*}(f)\right|_{g^{*} g}\right) \otimes g \quad \in K,
$$

where $f \in A=\operatorname{Ind}_{H^{\prime}}^{G}(D)$ and $\left.f\right|_{g} \in A$ denotes the function $\left.f\right|_{g}(k)=[k \equiv g] f(k)$ for all $k \in G_{H}$.

There is a $H^{\prime}$-equivariant epimorphism $\Phi: D \rightarrow A_{0}$ given by $\Phi(d)(t)=t^{*}(d)$ for $t \in H$ and $d \in D$. It is an isomorphism on the carrier algebra of $D$, so that $D \widehat{\rtimes} H^{\prime} \cong A_{0} \widehat{\rtimes} H^{\prime} \cong A_{0} \rtimes H \cong J$. Consequently we have obtained, by restricting to the ideals $I$ and $J$ in Theorem 3.7 and applying [18, Cor. 3.1], our result.

Acknowledgement. We thank the the Universidade Federal de Santa Catarina in Florianópolis for the support we received when developing the content of this paper in 2014. This note presents a slightly modified version (more detailed proofs) of a preprint in arXiv from 2014.

\section{REFERENCES}

[1] A. an Huef, I. Raeburn, and D. P. Williams. Proper actions on imprimitivity bimodules and decompositions of Morita equivalences. J. Funct. Anal., 200(2):401-428, 2003.

[2] P. Baum, A. Connes, and N. Higson. Classifying space for proper actions and $K$-theory of group $C^{*}$ - algebras. Contemp. Math. 167, 241-291 (1994).

[3] P. Baum, E. Guentner, and E. Willett. Expanders, exact crossed products, and the BaumConnes conjecture. Ann. K-theory, 1(2):155-208, 2016.

[4] B. Burgstaller. A note on a certain Baum-Connes map for inverse semigroups. preprint arXiv:1609.01913.

[5] B. Burgstaller. Attempts to define a Baum-Connes map via localization of categories for inverse semigroups. preprint arXiv:1506.08412, under review in Mathematical Reports.

[6] B. Burgstaller. A descent homomorphism for semimultiplicative sets. Rocky Mt. J. Math., 44(3):809-851, 2014.

[7] S. Echterhoff, S. Kaliszewski, J. Quigg, and I. Raeburn. A categorical approach to imprimitivity theorems of $C^{*}$-dynamical systems. Mem. Am. Math. Soc., 850, 2006.

[8] P. Green. The local structure of twisted covariance algebras. Acta Math., 140:191-250, 1978.

[9] G. G. Kasparov. Equivariant $K K$-theory and the Novikov conjecture. Invent. Math., 91:147201, 1988.

[10] G.G. Kasparov. $K$-theory, group $C^{*}$-algebras, and higher signatures (conspectus). In Novikov conjectures, index theorems and rigidity. Vol. 1., pages 101-146. Cambridge University Press, 1995.

[11] M. Khoshkam and G. Skandalis. Crossed products of $C^{*}$-algebras by groupoids and inverse semigroups. J. Oper. Theory, 51(2):255-279, 2004.

[12] E.C. Lance. Hilbert $C^{*}$-modules. A toolkit for operator algebraists. Cambridge: Univ. Press, 1995.

[13] R. Meyer and R. Nest. The Baum-Connes conjecture via localisation of categories. Topology, 45(2):209-259, 2006.

[14] A.L.T. Paterson. Groupoids, inverse semigroups, and their operator algebras. Progress in Mathematics (Boston, Mass.). 170. Boston, MA: Birkhäuser., 1999.

[15] J. Quigg and N. Sieben. $C^{*}$-actions of $r$-discrete groupoids and inverse semigroups. J. Aust. Math. Soc., Ser. A, 66(2):143-167, 1999.

[16] I. Raeburn. Induced $C^{*}$-algebras and a symmetric imprimitivity theorem. Math. Ann., 280(3):369-387, 1988.

[17] M. A. Rieffel. Induced representations of C*-algebras. Adv. Math., 13:176-257, 1974.

[18] M. A. Rieffel. Unitary representations of group extensions; an algebraic approach to the theory of Mackey and Blattner. Studies in analysis, Adv. Math., suppl. Stud., Vol. 4, 33-82 (1979)., 1979. 
[19] N. Sieben. $C^{*}$-crossed products by partial actions and actions of inverse semigroups. J. Aust. Math. Soc., Ser. A, 63(1):32-46, 1997.

[20] S. Vaes. A new approach to induction and imprimitivity results. J. Funct. Anal., 229(2):317374,2005

Departamento de Matematica, Universidade Federal de Santa Catarina, CeP 88.040900 FloRianóPOLIS-SC, BRASIL

E-mail address: bernhardburgstaller@yahoo.de 